\title{
Secondary Healthcare Payer
}

National Cancer Institute

\section{Source}

National Cancer Institute. Secondary Healthcare Paver. NCI Thesaurus. Code C70690.

A health plan or payer not responsible for being the primary payer on a claim for medical services. 